PROCEEDINGS OF THE

AMERICAN MATHEMATICAL SOCIETY

Volume 132, Number 3, Pages 649-658

S 0002-9939(03)07135-1

Article electronically published on July 31, 2003

\title{
BILIAISON CLASSES OF CURVES IN $\mathbf{P}^{3}$
}

\author{
ROSARIO STRANO \\ (Communicated by Bernd Ulrich)
}

\begin{abstract}
We characterize the curves in $\mathbf{P}^{3}$ that are minimal in their biliaison class. Such curves are exactly the curves that do not admit an elementary descending biliaison. As a consequence we have that every curve in $\mathbf{P}^{3}$ can be obtained from a minimal one by means of a finite sequence of ascending elementary biliaisons.
\end{abstract}

\section{INTRODUCTION}

Let $C$ be a curve (i.e., a locally Cohen-Macaulay subscheme equidimensional of dimension 1) in $\mathbf{P}=\mathbf{P}_{\mathbf{k}}^{3}$ over an algebraically closed field $\mathbf{k}$. Let $\mathcal{J}_{C}$ be its ideal sheaf in $\mathcal{O}_{\mathbf{P}}$ and $I_{C}=H_{*}^{0}\left(\mathcal{J}_{C}\right)$ be the homogeneous ideal of $C$ in the polynomial ring $R=\mathbf{k}\left[x_{0}, \ldots, x_{3}\right]=H_{*}^{0}\left(\mathcal{O}_{\mathbf{P}}\right)$. Use the notation

$$
\begin{gathered}
s(C)=\inf \left\{n \in \mathbf{Z} \mid h^{0}\left(\mathcal{J}_{C}(n)\right) \neq 0\right\} ; \\
e(C)=\sup \left\{n \in \mathbf{Z} \mid h^{1}\left(\mathcal{O}_{C}(n)\right) \neq 0\right\} .
\end{gathered}
$$

In 9 P. Rao introduced the notion of a biliaison (even linkage) class of curves and proved that two curves $C$ and $C^{\prime}$ are in the same class if and only if $H_{*}^{1}\left(\mathcal{J}_{C}\right) \cong$ $H_{*}^{1}\left(\mathcal{J}_{C^{\prime}}\right)(m)$ for some $m \in \mathbf{Z}$.

Before stating the main results of the paper we recall some basic notions concerning biliaison. A good reference for the subject is [6] or [8. Recall that two curves $C$ and $C^{\prime}$ are in the same biliaison class if there is an even number of linkages connecting them.

Definition 1. A non-ACM curve $C$ is said to be minimal (in its biliaison class) if, for every $C^{\prime}$ in the same biliaison class, $H_{*}^{1}\left(\mathcal{J}_{C}\right) \cong H_{*}^{1}\left(\mathcal{J}_{C^{\prime}}\right)(m)$ with $m \geq 0$.

Definition 2. Let $C$ be a curve and let $Q$ be a surface containing it. A curve $C^{\prime}$ is said to be obtained from $C$ by an elementary biliaison on $Q$ of height $m$ if there are surfaces $T$ and $V$ of degrees $t, t+m$, respectively, such that $C$ is linked to a curve $D$ by means of $(Q, T)$ and $D$ is linked to $C^{\prime}$ by means of $(Q, V)$.

The elementary biliaison is said to be ascending if $m>0$ and descending, if $m<0$. In particular, if $m>0$ and $T$ is a component of $V$, the elementary biliaison is said to be an elementary trivial biliaison or a basic double link of height $m$.

Received by the editors June 10, 2002 and, in revised form, October 25, 2002

2000 Mathematics Subject Classification. Primary 14H50; Secondary 14H45.

Key words and phrases. Space curves, liaison, elementary biliaison, minimal curves.

This work was done with the financial support of the MIUR (Italian Research Ministry).

(C)2003 American Mathematical Society 
In [3] R. Lazarsfeld and P. Rao proved that curves with $s(C) \geq e(C)+4$ are minimal in their biliaison class. In 6] M. Martin-Deschamps and D. Perrin gave a construction of the minimal curves in each biliaison class starting from the minimal free graded resolution of the Rao module $M(C)=H_{*}^{1}\left(\mathcal{J}_{C}\right)$.

In this paper, by completing the result of Lazarsfeld and Rao, we give a characterization of minimal non-ACM curves in the case $s(C) \leq e(C)+3$. These curves are those satisfying the following condition: $\left(^{*}\right)$ for every $s(C) \leq s \leq e(C)+3$ there is a form $H_{s}$ of positive degree $<s$ dividing every element in $H^{0}\left(\mathcal{J}_{C}(s)\right)$ and such that $H_{s} \cdot H^{0}\left(\omega_{C}(3-s)\right)=0$.

We also see that these curves are exactly the curves that do not admit any elementary descending biliaison; from this one can deduce the following theorem about the structure of a given biliaison class.

Every curve in $\mathbf{P}^{3}$ can be obtained from a minimal one by means of a finite number of ascending elementary biliaisons.

The paper is organized as follows: in Sections 1 and 2 we prove the main result (Theorem 1) and precisely in Section 1 we prove that a non-ACM curve with $s(C) \leq$ $e(C)+3$ that does not admit any elementary descending biliaison satisfies condition $\left(^{*}\right)$, while in Section 2 we prove that such a curve is minimal.

In Section 3 we deduce the above result (Theorem 2) regarding the structure of a given biliaison class. We also show that this result is a slight improvement of the Lazarsfeld-Rao property proved by M. Martin-Deschamps and Perrin [6] and by E. Ballico, G. Bolondi and J. Migliore 2, since it gives more control over the graded Betti numbers of a free resolution of $I_{C}$ (see [8] for the definition of the Lazarsfeld-Rao property).

As a final application (Theorem 3) we deduce a bound for the minimal number of generators of the homogeneous ideal $I_{C}$. This bound reduces to the Dubreil bound when $C$ is ACM and to the Amasaki bound when $C$ is arithmetically Buchsbaum.

We note that all the results of this paper can be easily generalized to codimension 2 subschemes (equidimensional and locally Cohen-Macaulay) in $\mathbf{P}^{n}$.

By abuse of notation we will denote with the same symbol a homogeneous polynomial in $R$ and the surface of $\mathbf{P}^{3}$ that it defines.

\section{1}

In this section first we state the main result of the paper. The notation is as in the Introduction. In order to include all possible curves in $\mathbf{P}^{3}$ we consider a line as a minimal curve in the biliaison class of the ACM curves in $\mathbf{P}^{3}$.

Theorem 1. For a curve $C \subset \mathbf{P}^{3}$ the following are equivalent:

1) $C$ is minimal in its biliaison class;

2) $C$ does not admit any elementary descending biliaison;

3) $C$ is of one (and only one) of the following types:

a) a line,

b) $s(C) \geq e(C)+4$,

c) $s(C) \leq e(C)+3$, and

(*) for every $s(C) \leq s \leq e(C)+3$ there is a form $H_{s}$ of positive degree $<s$ dividing every element in $H^{0}\left(\mathcal{J}_{C}(s)\right)$ and such that $H_{s} \cdot H^{0}\left(\omega_{C}(3-s)\right)=0$.

It is obvious that 1 ) implies 2). In the rest of this section we prove that 2) implies 3). The proof that 3) implies 1) will be done in the next section. First 
we prove some preliminary results. Recall that, for every $Q \in H^{0}\left(\mathcal{J}_{C}(s)\right)$ and for every $m \in \mathbf{Z}$, we have an exact sequence

$$
0 \rightarrow H^{0}\left(\mathcal{O}_{Q}(m)\right) \rightarrow \operatorname{Hom}\left(\mathcal{J}_{C / Q}, \mathcal{O}_{Q}(m)\right) \rightarrow H^{0}\left(\omega_{C}(4-s+m)\right) \rightarrow 0
$$

(see [6], Lemme III.2.4).

In particular, for $m=-1$ we have an isomorphism: $\operatorname{Hom}\left(\mathcal{J}_{C / Q}, \mathcal{O}_{Q}(-1)\right) \cong$ $H^{0}\left(\omega_{C}(3-s)\right)$.

The next proposition is essentially a reformulation of [10, Proposition 2.2.3 and its proof. (See [5] for another proof.)

Proposition 1. Let $s(C) \leq s \leq e(C)+3, Q \in H^{0}\left(\mathcal{J}_{C}(s)\right), \xi \in H^{0}\left(\omega_{C}(3-s)\right)$, $\xi \neq 0$ and let $\eta: \mathcal{J}_{C / Q} \rightarrow \mathcal{O}_{Q}(-1)$ be the morphism of $\mathcal{O}_{Q}$-modules corresponding to $\xi$. Then $\eta$ is not injective if and only if there is a form $H$ of degree $<s$ dividing $Q$ and such that $H \cdot \xi=0$.

Proof of Proposition 1. Assume that $\eta$ is not injective. By [10], Proposition 2.2.3, its kernel and its image are respectively of the form $\mathcal{J}_{D / Q}$ and $\mathcal{J}_{E / Q}(-1)$, where $D$, $E$ are subschemes of $Q$ containing respectively surfaces $H, K$ of degree $<s$ such that $H \cdot K=Q$. Since $\mathcal{J}_{E / Q}(-1) \subseteq \mathcal{J}_{K / Q}(-1)$ and $H$ kills $\mathcal{J}_{K / Q}(-1)$ we have $H \cdot \eta=0$ and hence $H \cdot \xi=0$. Conversely assume that there is a form $H$ of degree $<s$ dividing $Q$ and such that $H \cdot \xi=0$ and denote by $u: I_{C / Q} \rightarrow R_{Q}(-1)$ the (degree zero) homomorphism of graded $R$-modules corresponding to $\eta$. From the exact sequence

$$
0 \rightarrow H^{0}\left(\mathcal{O}_{Q}(h-1)\right) \rightarrow \operatorname{Hom}\left(\mathcal{J}_{C / Q}, \mathcal{O}_{Q}(-1)\right)(h) \rightarrow H^{0}\left(\omega_{C}(3-s+h)\right) \rightarrow 0
$$

where $h$ is the degree of $H$, we see that $H \eta \in H^{0}\left(\mathcal{O}_{Q}(h-1)\right)$. It follows that, if $Q^{\prime} \in I_{C}$ is a surface without common components with $Q$, we have $H u\left(Q^{\prime}\right)=S Q^{\prime}$ $(\bmod Q)$, where $S$ is a form of degree $h-1$. Since $H \mid Q$ we have $H \mid S Q^{\prime}$, but $H$ and $Q^{\prime}$ do not have common components and $\operatorname{deg} S<\operatorname{deg} H$. It follows that $S=0$. Hence $H \eta=0$ and hence the image of $\eta$ is of the form $\mathcal{J}_{E / Q}(-1)$, where $E \subset Q$ contains the surface $K$ where $H \cdot K=Q$. By [6], Proposition III.2.6, $\eta$ is not injective.

Remark 1. Fix $s$ and $\xi \neq 0$. For $Q \in H^{0}\left(\mathcal{J}_{C}(s)\right)$, if the corresponding morphism $\left.\eta: \mathcal{J}_{C / Q} \rightarrow \mathcal{O}_{Q}(-1)\right)$ is not injective we will denote by $H_{Q}$ the surface contained in $Q$ defined as the 2-dimensional component of the subscheme $D \subset Q$ whose ideal sheaf is ker $\eta$; we note that $H_{Q}$ can be characterized as follows: $H_{Q} \mid Q, H_{Q} \cdot \xi=0$ and for every $H^{\prime}$ s.t. $H^{\prime} \mid Q$ and $H^{\prime} \cdot \xi=0$ we have $H_{Q} \mid H^{\prime}$. In fact from the above result we have $H^{\prime} \cdot \eta=0$ and hence the image of $\eta$ is of the form $\mathcal{J}_{E / Q}(-1)$, where $E \subset Q$ contains the surface $K^{\prime}$ with $H^{\prime} \cdot K^{\prime}=Q$. Since $K$ is the 2-dimensional component of $E$ we have $K^{\prime} \mid K$ and hence $H=H_{Q}$ divides $H^{\prime}$.

Proposition 2. Let $s(C) \leq s \leq e(C)+3, Q, Q^{\prime} \in H^{0}\left(\mathcal{J}_{C}(s)\right), \xi \in H^{0}\left(\omega_{C}(3-s)\right)$, $\xi \neq 0$ and let $\eta: \mathcal{J}_{C / Q} \rightarrow \mathcal{O}_{Q}(-1)$ and $\eta^{\prime}: \mathcal{J}_{C / Q^{\prime}} \rightarrow \mathcal{O}_{Q}^{\prime}(-1)$ be the morphisms corresponding to $\xi$. Assume that $\eta$ and $\eta^{\prime}$ are not injective and let $H_{Q}$ and $H_{Q^{\prime}}$ be the surfaces defined above. Then $H_{Q}=H_{Q^{\prime}}$.

Proof of Proposition 2. Put $H=H_{Q}$ and $H^{\prime}=H_{Q^{\prime}}$. Assume that they have degrees $0<h \leq h^{\prime}<s$. Since $H \cdot \xi=0$ and $H^{\prime} \cdot \xi=0$ it is enough to prove $H \mid H^{\prime}$. In fact, in this case $H \mid Q^{\prime}$ and by Remark $1, H^{\prime} \mid H$.

Since $H^{\prime} \cdot \xi=0$ we have $H^{\prime} \cdot u\left(Q_{i}\right)=S Q_{i}(\bmod Q)$ for all $Q_{i} \in I_{C}$, where $S$ is a surface of degree $h^{\prime}-1$ and $u$ is as before. From this we get $K \mid S$ since the image 
$u\left(I_{C / Q}\right)$ is contained in the ideal $I_{E / Q}(-1)$ and hence all $u\left(Q_{i}\right)$ are multiples of $K$. In particular, for $Q_{i}=Q^{\prime}$, putting $u\left(Q^{\prime}\right)=K T, S=K V$ we have $H^{\prime} T=H^{\prime} K^{\prime} V$ $(\bmod H)$, i.e., $H^{\prime} T=H^{\prime} K^{\prime} V+Z H$ with $Z$ of degree $h^{\prime}-1$. It follows that $H^{\prime}$ divides $Z H$, and hence $H^{\prime}$ has a common factor of positive degree with $H$.

Let $F=G C D\left(H, H^{\prime}\right)$; we will show that $F=H$. Assume the contrary, i.e., $\operatorname{deg} F<h$ and put $H=F \cdot \bar{H}, Q=F \cdot \bar{Q}=F \cdot \bar{H} \cdot K$ and similarly $H^{\prime}=F \cdot \overline{H^{\prime}}$, $Q^{\prime}=F \cdot \overline{Q^{\prime}}=F \cdot \overline{H^{\prime}} \cdot K^{\prime}$.

Now let $Y$ be the residual curve of $C$ with respect to $F$, i.e., the curve whose homogeneous ideal is $\left(I_{C}: F\right)$. It is easy to see that $\left(I_{C}: F\right)$ is a saturated ideal and that $Y \subset C$ is a curve i.e., does not have zero-dimensional components (isolated or embedded). Moreover, $Y$ is not empty; in fact if it were, $F \in I_{C}$ and we would have $F \cdot \xi=0$ and this is contrary to the fact that $H$ is the surface of minimal degree contained in $Q$ such that $H \cdot \xi=0$.

From the exact sequence

$$
0 \rightarrow \mathcal{O}_{Y}(-f) \rightarrow \mathcal{O}_{C} \rightarrow \mathcal{O}_{C \cap F} \rightarrow 0
$$

we have an exact sequence of sheaves on $C$

$$
0 \rightarrow \omega_{C \cap F} \rightarrow \omega_{C} \rightarrow \omega_{Y}(f)
$$

where $f=\operatorname{deg} F$. From the above we obtain an exact sequence

$$
0 \rightarrow H^{0}\left(\omega_{C \cap F}(3-s)\right) \rightarrow H^{0}\left(\omega_{C}(3-s)\right) \rightarrow H^{0}\left(\omega_{Y}(3-s+f)\right)
$$

and let $\bar{\xi}$ be the image of $\xi$ in $H^{0}\left(\omega_{Y}(3-s+f)\right)$. Hence we have:

1) $\bar{\xi} \neq 0$. In fact if it were zero, $\xi$ would be in $H^{0}\left(\omega_{C \cap F}(3-s)\right)$ and hence $F \cdot \xi=0$. It follows that $3-s+f \geq-e(Y)$.

2) $Y \subset \bar{Q}$ and $Y \subset \overline{Q^{\prime}}$. It follows that $s(Y) \leq s-f$.

3) $\bar{H}$ divides $\bar{Q}$ and has degree $0<h-f<s-f$; $\overline{H^{\prime}}$ divides $\overline{Q^{\prime}}$ and has degree $0<h^{\prime}-f<s-f$.

4) $\bar{H} \cdot \bar{\xi}=0$ and $\overline{H^{\prime}} \cdot \bar{\xi}=0$. In fact $H^{0}\left(\omega_{Y}(3-s+f)\right)$ embeds in $H^{0}\left(\omega_{C}(3-s+f)\right)$ and the composition $H^{0}\left(\omega_{C}(3-s)\right) \rightarrow H^{0}\left(\omega_{Y}(3-s+f)\right) \rightarrow H^{0}\left(\omega_{C}(3-s+f)\right)$ is the multiplication by $F$. Hence $H \cdot \xi=\bar{H} \cdot F \xi=0$ implies that $\bar{H} \cdot \bar{\xi}=0$ and similarly for $H^{\prime}$.

We have hence reproduced for $Y$ the hypotheses of Proposition 2, and by the first part of the proof, $\bar{H}$ and $\overline{H^{\prime}}$ have a common factor of positive degree and this is absurd since they were coprime.

Now we conclude the proof that 2) implies 3) in Theorem 1. Assume that $C$ does not admit any elementary descending biliaison of height -1 and $s(C) \leq e(C)+3$. Using [6], Proposition III.2.6, three cases are possible.

i) There is an $s, s(C) \leq s \leq e(C)+3$, a surface $Q$ of degree $s$ containing $C$ and a surjective homomorphism $\mathcal{J}_{C / Q} \rightarrow \mathcal{O}_{Q}(-1)$. In this case $C$ is the section of $Q$ with a plane $S$ and hence we can make a descending elementary biliaison of $C$ on $S$ with a line.

ii) There is an $s, s(C) \leq s \leq e(C)+3$, a surface $Q$ of degree $s$ containing $C$ and an injective non-surjective homomorphism $\mathcal{J}_{C / Q} \rightarrow \mathcal{O}_{Q}(-1)$. In this case $C$ admits an elementary descending biliaison on $Q$ (see [6], Prop. III.2.3).

iii) for all $s, s(C) \leq s \leq e(C)+3$ and all $Q$ of degree $s$, every nonzero homomorphism $\mathcal{J}_{C / Q} \rightarrow \mathcal{O}_{Q}(-1)$ is not injective. In this case, by Proposition 2, for every 
$s$ and every nonzero $\xi \in H^{0}\left(\omega_{C}(3-s)\right)$ there is a form of degree $<s$ that divides every element in $H^{0}\left(\mathcal{J}_{C}(s)\right)$ and such that $H \xi=0$.

The last step is to make $H$ independent of $\xi$. This is standard. Since for fixed $H$ the set of $\xi \in H^{0}\left(\omega_{C}(3-s)\right)$ s.t. $H \cdot \xi=0$ is a k-subspace of $H^{0}\left(\omega_{C}(3-s)\right)$ and since the set of forms of degree $<s$ dividing the GCD of $H^{0}\left(\mathcal{J}_{C}(s)\right)$ is finite, we have the result. This will finish our proof.

Remark 2. We note that $H$ divides all $H^{0}\left(\mathcal{J}_{C}(i)\right)$ for all $i=s(C), \ldots, s$ and kills all $H^{0}\left(\omega_{C}(j)\right)$ for $j=-e(C), \ldots, 3-s$. This last statement follows from the fact that there is a linear form in $R$ that is not a zero divisor for $H_{*}\left(\omega_{C}\right)$.

Remark 3. If $C$ is a curve satisfying condition 3)c) of Theorem 1 , then $C$ is not $\mathrm{ACM}$. In fact, an ACM curve has all its minimal generators in degree $\leq e(C)+3$; but for the condition 3)c), all these generators have a common factor of positive degree. As a consequence we have that an ACM curve of degree $>1$ always admits a descending elementary biliaison.

In this section we prove the implication 3$) \Rightarrow 1$ ) of Theorem 1 . Let $C$ be a curve satisfying condition 3)c); we want to prove that it is minimal in its biliaison class.

Let

$$
0 \rightarrow P \rightarrow N_{1} \rightarrow I_{C} \rightarrow 0
$$

be a minimal $N$-resolution of $I_{C}$ (see $[3$ or $[6$ ); we know that $P$ is a free graded $R$-module. Now let $C^{\prime}$ be another curve in the same biliaison class of $C$. By adding a free graded $R$-module $L$ we can assume that $C, C^{\prime}$ have $N$-resolutions (not necessarily minimal) of the form

$$
\begin{gathered}
0 \rightarrow A \stackrel{\alpha}{\longrightarrow} N \stackrel{\tau}{\longrightarrow} I_{C} \rightarrow 0, \\
0 \rightarrow B \stackrel{\beta}{\longrightarrow} N \stackrel{\sigma}{\longrightarrow} I_{C^{\prime}}(d) \rightarrow 0
\end{gathered}
$$

where $N=N_{1} \oplus L$,

$$
\begin{gathered}
A=\bigoplus_{i=1}^{n} R\left(-a_{i}\right)=P \oplus L, a_{1} \leq a_{2} \leq \cdots \leq a_{n}, \\
B=\bigoplus_{i=1}^{n} R\left(-b_{i}\right), b_{1} \leq b_{2} \leq \cdots \leq b_{n},
\end{gathered}
$$

and $d=\sum b_{i}-\sum a_{i}$.

As in [3], Section 2, Proof of Lemma 1.2, it is enough to prove that $b_{i} \geq a_{i}$ for all $i=1 \cdots n$. With the same notation as in $\left[3\right.$ it is enough to prove that rank $A_{\geq t} \geq$ rank $B_{\geq t}$ for all $t \in \mathbf{Z}$ where $A_{\geq t}=\bigoplus_{-a_{i} \geq t} R\left(-a_{i}\right)$ and $B_{\geq t}=\bigoplus_{-b_{i} \geq t} R\left(-b_{i}\right)$. We consider three cases:

1) $t \leq-(e(C)+4)$ : in this case the same proof as in $[3$ holds;

2) $t>-s(C)$ : in this case the same proof as in [3] holds;

3) let $-s(C) \geq t>-(e(C)+4)$ and assume that there exists $t$ such that $\operatorname{rank} A_{\geq t}<\operatorname{rank} B_{\geq t}$. We put $s=-t$ and we have $s(C) \leq s \leq e(C)+3$ and rank $A_{\geq-s}<$ rank $B_{\geq-s}$. We also assume that $s$ is minimal with this property.

Put $B=B_{1} \oplus B_{2}$ where $B_{1}=\bigoplus_{b_{i} \leq s} R\left(-b_{i}\right), B_{2}=\bigoplus_{b_{i}>s} R\left(-b_{i}\right)$ with ranks $r, n-r$, respectively, and similarly $A=A_{1} \bigoplus A_{2}$ where $A_{1}=\bigoplus_{a_{i} \leq s} R\left(-a_{i}\right)$, $A_{2}=\bigoplus_{a_{i}>s} R\left(-a_{i}\right)$ with ranks $r^{\prime}, n-r^{\prime}$, respectively, and $r>r^{\prime}$. 
Let $H=H_{s}$ be the form of degree $h<s$ given in 3)c) of Theorem 1; put $A^{\prime}=A_{1} \oplus A_{2}(h)$, and define $\phi: A \rightarrow A^{\prime}$ by $\phi: A_{1} \oplus A_{2} \rightarrow A_{1} \oplus A_{2}(h), \phi=1 \oplus H$.

Having fixed notation we prove three propositions. The aim of these propositions is to prove that the composition map $A_{1} \rightarrow N \rightarrow I_{C^{\prime}}(d)$ is zero.

Proposition 3. Under the above hypotheses and notation there is an exact sequence $0 \longrightarrow A_{1} \oplus A_{2}(h) \stackrel{\alpha^{\prime}}{\longrightarrow} M \oplus A_{2}(h) \stackrel{\tau^{\prime}}{\longrightarrow} I_{C} \longrightarrow 0$ and a commutative diagram

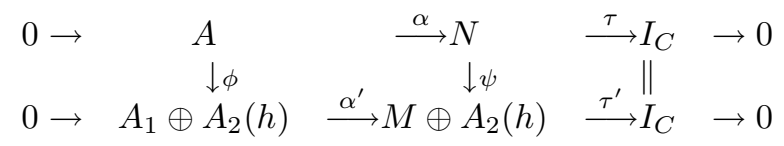

with $M$ a torsion free graded $R$-module of rank $r^{\prime}+1$ and where the map $\alpha^{\prime}$ is the direct sum of the identity on $A_{2}(h)$ and an inclusion $\theta: A_{1} \rightarrow M$.

Proof of Proposition 3. The proposition follows from the fact that, if $s<p$, then $\operatorname{Ext}^{1}\left(I_{C}, R(-p)\right)^{0} \cong H^{0}\left(\omega_{C}(4-p)\right)$ with $3-s \geq 4-p$ is killed by $H$. In more detail let $E \in \operatorname{Ext}^{1}\left(I_{C}, A\right)^{0}$ be the extension $0 \rightarrow A \rightarrow N \rightarrow I_{C} \rightarrow 0$ and let $\bar{E} \in \operatorname{Ext}^{1}\left(I_{C}, A_{2}\right)^{0}$ be the extension $0 \rightarrow A_{2} \rightarrow \bar{N} \rightarrow I_{C} \rightarrow 0$ induced by $E$, where $\bar{N}$ is the quotient of $N$ by $A_{1}$. Since $H$ kills $\bar{E}$ it follows that there is a map $\bar{N} \rightarrow A_{2}(h)$ such that the composition $A_{2} \rightarrow \bar{N} \rightarrow A_{2}(h)$ is the multiplication by $H$. Hence we get a map $N \rightarrow A_{2}(h)$ such that the composition $A_{2} \rightarrow N \rightarrow A_{2}(h)$ is the multiplication by $H$ and the composition $A_{1} \rightarrow N \rightarrow A_{2}(h)$ is zero. Now we consider the composite extension $\phi E \in \operatorname{Ext}^{1}\left(I_{C}, A^{\prime}\right)^{0}$ (see [4, Ch. III, Lemma 1.4). $\phi E$ has the form

$$
0 \rightarrow A_{1} \oplus A_{2}(h) \rightarrow N^{\prime} \rightarrow I_{C} \rightarrow 0,
$$

and we have a commutative diagram

$$
\begin{array}{ccccc}
0 \rightarrow & A & \stackrel{\alpha}{\longrightarrow} N & \stackrel{\tau}{\longrightarrow} I_{C} & \rightarrow 0 \\
& \downarrow_{\phi} & \downarrow & \downarrow \\
0 \rightarrow & A_{1} \oplus A_{2}(h) & \stackrel{\alpha^{\prime}}{\longrightarrow} N^{\prime} & \stackrel{\tau^{\prime}}{\longrightarrow} I_{C} & \rightarrow 0 .
\end{array}
$$

From the definition of $N^{\prime}$ we deduce that there is a map $N^{\prime} \rightarrow A_{2}(h)$ such that the composition $A_{2}(h) \rightarrow N^{\prime} \rightarrow A_{2}(h)$ is the identity and the composition $A_{1} \rightarrow$ $N^{\prime} \rightarrow A_{2}(h)$ is zero. Hence $N^{\prime}$ split as $M \oplus A_{2}(h)$, where $M=\operatorname{ker}\left(N^{\prime} \rightarrow A_{2}(h)\right)$ is a torsion free graded $R$-module of rank $r^{\prime}+1$; moreover, one sees that the map $\alpha^{\prime}$ is the direct sum of the identity on $A_{2}(h)$ and an inclusion $\theta: A_{1} \rightarrow M$.

Proposition 4. Under the above hypotheses and notation the composition

$$
B_{1} \stackrel{\beta_{1}}{\longrightarrow} N \stackrel{\psi}{\longrightarrow} N^{\prime} \stackrel{\pi}{\longrightarrow} A_{2}(h)
$$

is zero, where $\beta_{1}=\beta_{\mid B_{1}}$ and $\pi$ is the projection.

Proof of Proposition 4. A summand of $B_{1}$ is of the form $R(-b)$ with $b \leq s$. Since the terms in $A_{2}(h)$ have the form $R(-p+h), s-h<p-h$, hence $-p+h+b<h$, the map $R(-b) \rightarrow R(-p+h)$ will be zero if we can prove that it factors through the multiplication by $H$ or equivalently that restricted to the surface $H$ it is zero. To this end we first restrict to the affine open set $U=\mathbf{P}^{3} \backslash S$ where $S$ is a surface containing $C$ and without common components with $H$. Since $\mathcal{J}_{C, U} \cong \mathcal{O}_{U}$ the induced sequences

$$
\begin{array}{ccccc}
0 \rightarrow & \mathcal{A}_{U} & \longrightarrow \mathcal{N}_{U} & \rightarrow \mathcal{J}_{C, U} & \rightarrow 0 \\
& \downarrow \tilde{\phi}_{U} & & \| & \\
0 \rightarrow \tilde{\psi}_{U} & \mathcal{A}_{1, U} \oplus \mathcal{A}_{2}(h)_{U} & \rightarrow \mathcal{M}_{U} \oplus \mathcal{A}_{2}(h)_{U} & \rightarrow \mathcal{J}_{C, U} & \rightarrow 0
\end{array}
$$


split; hence they remain exact when restricted to $H \cap U$. Now the map $\mathcal{O}(-b)_{U} \rightarrow$ $\mathcal{J}_{C, U}$ induced by $R(-b) \rightarrow I_{C}$ is zero when restricted to $H \cap U$, since $b \leq s$ and $H$ divides all $H^{0}\left(\mathcal{J}_{C}(b)\right)$ for $b \leq s$. So the map $\mathcal{O}(-b)_{U} \rightarrow \mathcal{O}(-p+h)_{U}$ factors (mod $H \cap U)$ through $\mathcal{A}_{U}$, but the map $\mathcal{A}_{U} \rightarrow \mathcal{O}(-p+h)_{U}$ is zero $(\bmod H \cap U)$.

From Proposition 4 it follows that the map $\phi \circ \beta_{1}: B_{1} \rightarrow N^{\prime}$ factors through the inclusion $M \rightarrow N^{\prime}$ and since rank $B_{1}=r>r^{\prime}$ and rank $M=r^{\prime}+1$ it follows that rank $B_{1}=\operatorname{rank} M=r=r^{\prime}+1$.

Proposition 5. Under the above hypotheses and notation the composition map $\gamma: A_{1} \stackrel{\alpha_{1}}{\longrightarrow} N \stackrel{\sigma}{\longrightarrow} I_{C^{\prime}}(d)$ is zero.

Proof of Proposition 5. First we claim that the composition map

$$
N \stackrel{\sigma}{\longrightarrow} I_{C^{\prime}}(d) \stackrel{\cdot H}{\longrightarrow} I_{C^{\prime}}(d+h)
$$

factors through the map $\psi: N \rightarrow N^{\prime}$.

In fact, coker $\psi$ is isomorphic to coker $\phi$; hence it is annihilated by $H$. From the exact sequence

$$
\operatorname{Hom}\left(N^{\prime}, I_{C^{\prime}}(d)\right) \rightarrow \operatorname{Hom}\left(N, I_{C^{\prime}}(d)\right) \rightarrow \operatorname{Ext}^{1}\left(\operatorname{coker} \psi, I_{C^{\prime}}(d)\right)
$$

we get the claim since $H$ annihilates $\operatorname{Ext}^{1}\left(\operatorname{coker} \psi, I_{C^{\prime}}(d)\right)$.

Now letting $\sigma^{\prime}: N^{\prime} \rightarrow I_{C^{\prime}}(d+h)$ be the induced map, we prove that $\sigma_{\mid M}^{\prime}: M \rightarrow$ $I_{C^{\prime}}(d+h)$ is zero. In fact, its kernel contains $B_{1}$, hence has $\operatorname{rank} r=\operatorname{rank} M$, and its image is contained in $I_{C^{\prime}}(d+h)$, which is torsion free.

Now we consider the composition of $\gamma$ with the multiplication map $I_{C^{\prime}}(d) \stackrel{\cdot H}{\longrightarrow}$ $I_{C^{\prime}}(d+h)$. It factors through the map $\sigma_{\mid M}^{\prime}: M \rightarrow I_{C^{\prime}}(d+h)$; hence it is zero and $\gamma$ is also zero.

From Proposition 5 we have that the map $\alpha_{1}: A_{1} \rightarrow N$ factors as $\beta_{1} \circ \zeta$ where $\zeta: A_{1} \rightarrow B_{1}$ and $\beta_{1}: B_{1} \rightarrow N$. It follows that, for every $c \leq s, \zeta$ induces an inclusion

$$
\bigoplus_{a_{i} \leq c} R\left(-a_{i}\right) \hookrightarrow \bigoplus_{b_{i} \leq c} R\left(-b_{i}\right)
$$

Hence for every $c \leq s$ we have rank $A_{\geq-c} \leq \operatorname{rank} B_{\geq-c}$.

On the other hand, we remember that $s$ was the minimum such that rank $A_{\geq-s}<$ rank $B_{\geq-s}$, hence for $c<s$ is rank $A_{\geq-c} \geq \operatorname{rank} B_{\geq-c}$. It follows that for every $c<s, \sharp\left\{a_{i}=c\right\}=\sharp\left\{b_{i}=c\right\}$ and $\zeta$ induces an isomorphism

$$
\bigoplus_{a_{i} \leq c} R\left(-a_{i}\right) \cong \bigoplus_{b_{i} \leq c} R\left(-b_{i}\right)
$$

Moreover, since rank $A_{1}+1=\operatorname{rank} B_{1}$, we have that for $c=s$ we can change the basis in $\bigoplus_{b_{i}=s} R\left(-b_{i}\right)$ such that $\bigoplus_{b_{i}=s} R\left(-b_{i}\right)=\bigoplus_{a_{i}=s} R\left(-a_{i}\right) \oplus R(-s)$ and $\zeta$ induces the identity on $\bigoplus_{a_{i}=s} A\left(-a_{i}\right)$.

In conclusion we have a split exact sequence

$$
0 \rightarrow A_{1} \stackrel{\zeta}{\longrightarrow} B_{1} \rightarrow R(-s) \rightarrow 0 .
$$

We then can factor out $N$ by $A_{1}$, and we can assume in the sequel that $A_{1}=0$ and $B_{1}=R(-s), M=I_{C}$.

The last part of the proof is now aiming to get a contradiction by showing that the map $\beta: B \rightarrow N$, whose cokernel is $I_{C^{\prime}}(d)$, drops rank on a surface $K$ of $\mathbf{P}^{3}$, thus contradicting the Hilbert-Burch theorem. 
Let in fact $Q=H \cdot K$ be the image of the generator of $R(-s)$ in $I_{C}$. As in the proof of Proposition 4 we restrict to the affine open set $U=\mathbf{P}^{3} \backslash S$ where $S$ is a surface containing $C$ and without common components with $Q$. Moreover, we still denote by $Q, H, K$ the local equations of the surfaces $Q, H, K$ on $U$.

Since $\mathcal{J}_{C, U} \cong \mathcal{O}_{U}$ the induced sequences $0 \rightarrow \mathcal{A}_{U} \rightarrow \mathcal{N}_{U} \rightarrow \mathcal{J}_{C, U} \rightarrow 0$ and $0 \rightarrow \mathcal{A}_{U}^{\prime} \rightarrow \mathcal{N}_{U}^{\prime} \rightarrow \mathcal{J}_{C, U} \rightarrow 0$ split. Hence $\mathcal{N}_{U} \cong \mathcal{A}_{U} \oplus \mathcal{J}_{C, U} \cong \mathcal{O}_{U}^{\oplus n} \oplus \mathcal{O}_{U}$ and $\mathcal{N}_{U}^{\prime} \cong \mathcal{A}_{U}^{\prime} \oplus \mathcal{J}_{C, U} \cong \mathcal{O}_{U}^{\oplus n} \oplus \mathcal{O}_{U}$. We write the matrix associated to the map $\tilde{\psi}_{U}: \mathcal{O}_{U}^{\oplus n+1} \rightarrow \mathcal{O}_{U}^{\oplus n+1}$ induced by $\psi$. It has the form

$$
\mathbf{M}={\mathcal{\mathcal { A } _ { U } ^ { \prime }}}_{\mathcal{J}_{C, U}\{}\left\{\left(\begin{array}{cc}
\overbrace{H \mathrm{I}_{n}}^{\mathcal{A}_{U}} & \overbrace{\mathrm{C}_{n}}^{\mathcal{J}_{C, U}} \\
0 & 1
\end{array}\right)\right.
$$

where $\mathrm{I}_{n}$ is the unit matrix and $\mathrm{C}_{n}$ is a one-column matrix.

We also consider the matrix associated to the map $\tilde{\beta}_{U}: \mathcal{B}_{U} \cong \mathcal{O}(-s)_{U} \oplus \mathcal{B}_{2, U} \rightarrow$ $\mathcal{N}_{U} \cong \mathcal{A}_{U} \oplus \mathcal{J}_{C, U}$ induced by the map $\beta: B \rightarrow N$. It has the form

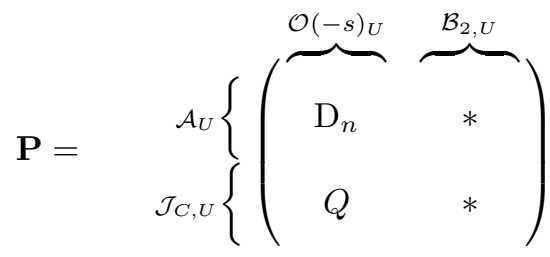

where $\mathrm{D}_{n}$ is a one-column matrix.

The product $\mathbf{M} \cdot \mathbf{P}$ is associated to the map $\tilde{\phi}_{U} \circ \tilde{\beta}_{U}: \mathcal{B}_{U} \rightarrow \mathcal{N}_{U}^{\prime} \cong \mathcal{A}_{U}^{\prime} \oplus \mathcal{J}_{C, U}$ and has the form

$$
\mathbf{M} \cdot \mathbf{P}={\mathcal{\mathcal { A } _ { U } ^ { \prime }}}_{\mathcal{J}_{C, U}\{}\left\{\left(\begin{array}{cc}
H \overbrace{\mathrm{D}_{n}+Q \mathrm{C}_{n}}^{\mathcal{O}(-s)_{U}} & * \\
Q & *
\end{array}\right) .\right.
$$

By Proposition 4 we have $H \mathrm{D}_{n}+Q \mathrm{C}_{n}=0$, hence $\mathrm{D}_{n}=-K \mathrm{C}_{n}$, and the matrix $\mathbf{P}$ has the form

$$
\mathbf{P}=\mathcal{A}_{U}\left\{\left(\begin{array}{cc}
\overbrace{\mathcal{J}_{C, U}}\left\{\mathrm{C}_{n}\right. & * \\
Q & *
\end{array}\right) .\right.
$$

We finish by observing that all $n \times n$ minors of $\mathbf{P}$ are a multiple of $K$ and this is contrary to the Hilbert-Burch theorem.

Remark 4. From Theorem 1 it follows that a minimal curve that is integral either is a line or has $s(C) \geq e(C)+4$. 
In this section we deduce some consequences from Theorem 1. The first consequence, whose proof is trivial, is the following theorem about the structure of a biliaison class.

Theorem 2. Every curve in $\mathbf{P}^{3}$ can be obtained from a minimal one by means of a finite number of ascending elementary biliaisons.

Remark 5. From the proof of Theorem 1 we see that a curve that is not minimal admits an elementary biliaison of height -1 ; hence we can conclude that every curve in $\mathbf{P}^{3}$ can be obtained from a minimal one by means of a finite number of ascending elementary biliaisons of height 1 .

We now show that Theorem 2 is a slight improvement of the Lazarsfeld-Rao property proved by Martin-Deschamps and Perrin [6] and by Ballico, Bolondi and Migliore [2], since it gives more control on the Betti numbers of a free resolution of $I_{C}$ (see 8 for the definition of the Lazarsfeld-Rao property).

Corollary 1. Every curve $C$ in $\mathbf{P}^{3}$ can be obtained from a minimal one $C_{0}$ by means of a finite number of basic double links $C_{0}, C_{1}^{\prime}, \ldots, C_{t}^{\prime}=C^{\prime}$ followed by a generalizing deformation, i.e., a deformation such that, for all curves of the family, the graded Betti numbers are not greater than the corresponding graded Betti numbers of $C^{\prime}$.

Proof of Corollary 1. By Remark 5 we have a sequence of elementary ascending biliaisons of height $1, C_{0}, C_{1}, \ldots, C_{t}=C$ on surfaces of degrees $s_{1}, \ldots, s_{t}$. We claim that there is a sequence of basic double links of height $1, C_{0}, C_{1}^{\prime}, \ldots, C_{t}^{\prime}=C^{\prime}$ on surfaces of degrees $s_{1}, \ldots, s_{t}$ such that the curves $C$ and $C^{\prime}$ have minimal $E$ resolutions of the form $0 \rightarrow E \rightarrow F \rightarrow I_{C} \rightarrow 0$ and $0 \rightarrow E^{\prime} \rightarrow F^{\prime} \rightarrow I_{C^{\prime}} \rightarrow 0$ respectively, where $F, F^{\prime}$ are free $R$-modules, with $E \oplus L \cong E^{\prime}$ and $F \oplus L \cong F^{\prime}$, for some free $R$-module $L$.

In fact, by induction on $i$, let us assume that $C_{i}$ and $C_{i}^{\prime}$ have minimal $E$ resolutions of the form $0 \rightarrow E_{i} \rightarrow F_{i} \rightarrow I_{C_{i}} \rightarrow 0$ and $0 \rightarrow E_{i}^{\prime} \rightarrow F_{i}^{\prime} \rightarrow I_{C_{i}^{\prime}} \rightarrow 0$, respectively, where $F_{i}, F_{i}^{\prime}$ are free $R$-modules, with $E_{i} \oplus L_{i} \cong E_{i}^{\prime}$ and $F_{i} \oplus L_{i} \cong F_{i}^{\prime}$, for some free $R$-module $L_{i}$. Let $C_{i+1}$ be obtained from $C_{i}$ by an elementary biliaison of height 1 on a surface $Q_{i+1}$ of degree $s_{i+1}$. Two cases are possible:

a) $Q_{i+1}$ is a minimal generator of $I_{C_{i}}$. In this case $F_{i}$ contains a direct summand $R\left(-s_{i+1}\right)$ and hence we can choose a minimal generator $Q_{i+1}^{\prime}$ of $I_{C_{i}^{\prime}}$ of degree $s_{i+1}$.

b) $Q_{i+1}$ is not a minimal generator of $I_{C_{i}}$, i.e., $Q_{i+1}=\sum M_{l} G_{l}$ with $G_{l}$ minimal generators of degree strictly less than $s_{i+1}$. In this case we can do the same for $C_{i}^{\prime}$ and choose a surface $Q_{i+1}^{\prime}=\sum M_{l} G_{l}^{\prime}$, with $G_{l}^{\prime}$ minimal generators $I_{C_{i}^{\prime}}$ of the same degree of $G_{l}$.

We perform a basic double link of $C_{i}^{\prime}$ on $Q_{i+1}^{\prime}$ of height 1. Using [6], Prop. III.4.3 and the successive proposition 4.5 , we easily get the claim.

Now if we add $L$ to the terms of the $E$-resolution of $C$, by arguing as in [8], Proposition 6.3.3, we get the required family.

Remark 6. With the notation as in Corollary 1 we see that the graded Betti numbers of $C$ are obtained from those of $C^{\prime}$ by making (possibly) a cancellation of a common free direct summand between the generators and the first syzygies, the second syzygies remaining unaltered. 
In [7] R. Maggioni and A. Ragusa proved an upper bound for the graded Betti numbers of the homogeneous ideal $I_{C}$ for curves obtained from a minimal one by a finite number of basic double links, in terms of the Hilbert function. As a consequence of Corollary 1 this upper bound holds for all curves in $\mathbf{P}^{3}$.

Finally we prove a bound for the minimal number of generators of the homogeneous ideal $I_{C}$.

Theorem 3. Let $C$ be any curve in $\mathbf{P}^{3}$ and let $C_{0}$ be a minimal curve in the biliaison class of $C$. Let us denote by $\nu(C), \nu\left(C_{0}\right)$ the minimal number of generators of $I_{C}, I_{C_{0}}$, respectively. Then we have

$$
\nu(C) \leq \nu\left(C_{0}\right)+s(C)-s\left(C_{0}\right)
$$

Proof. Using [6], Prop. III.4.3, it is easy to see that, if $C$ is obtained from $C^{\prime}$ by means of an elementary biliaison of height 1 on a surface $Q$ of degree $s$, then two cases are possible:

a) $s=s\left(C^{\prime}\right)$ : in this case $s(C)=s\left(C^{\prime}\right)$ and $C, C^{\prime}$ have the same number of minimal generators;

b) $s>s\left(C^{\prime}\right)$ : in this case $s(C)=s\left(C^{\prime}\right)+1$ and $C$ has at most one more minimal generator than $C^{\prime}$, i.e., the surface $Q$.

We apply Theorem 3 to the following cases:

1) $C$ is an ACM curve. In this case $C_{0}$ is a line and we get the well-known Dubreil bound $\nu(C) \leq s(C)+1$.

2) $C$ is an arithmetically Buchsbaum curve and let $\mu=\operatorname{dim}_{\mathbf{k}} M(C)$. In this case we have $s\left(C_{0}\right)=2 \mu$ and $\nu\left(C_{0}\right)=3 \mu+1$ (see e.g. [6]) and we get the Amasaki bound $\nu(C) \leq s(C)+\mu+1$ (see [1]).

\section{REFERENCES}

[1] M. Amasaki, On the structure of arithmetically Buchsbaum curves in $\mathbf{P}_{k}^{3}$, Publ. Res. Inst. Math. Sci. 20 (1984), 793-837. MR 86a:14027

[2] E. Ballico, G. Bolondi, and J. C. Migliore, The Lazarsfeld-Rao problem for liaison classes of two-codimensional subschemes of $\mathbf{P}^{n}$, Amer. J. Math. 113 (1991), 117-128. MR 92c:14047

[3] R. Lazarsfeld and A. P. Rao, Linkage of general curves of large degree, Lecture Notes in Math. 997 (1983). MR 85d:14043

[4] S. Mac Lane, Homology, Grundlehren der mathematischen Wissenschaften, Band 114, Springer-Verlag, Berlin, 1967. MR 50:2285

[5] M. Martin-Deschamps, Minimalité des courbes sous-canoniques, Ann. Inst. Fourier (Grenoble) $\mathbf{5 2}$ (2002), 1027-1040.

[6] M. Martin-Deschamps and D. Perrin, Sur la classification des courbes gauches, Astérisque 184-185 (1990). MR 91h:14039

[7] R. Maggioni and A. Ragusa, Betti numbers of space curves bounded by Hilbert functions, Le Matematiche 52 (1997), 217-232. MR 99e:14035

[8] J. C. Migliore, Introduction to Liaison Theory and Deficiency Modules, Birkhäuser, Boston, 1998. MR 2000g:14058

[9] A. P. Rao, Liaison among curves in $\mathbf{P}^{3}$, Inventiones Math. 50 (1979), 205-217. MR 80e: 14023

[10] E. Schlesinger, The spectrum of projective curves, Ph.D. Thesis, U. C. Berkeley, 1996.

Department of Mathematics and Informatics, University of Catania, Viale A. Doria 6, I95125 Catania, Italy

E-mail address: sstrano@dmi.unict.it 